\title{
Designing Movement-based Play With Young People Using Powered Wheelchairs
}

\author{
Kathrin Gerling ${ }^{1}$, Kieran Hickss ${ }^{1}$, Michael Kalyn ${ }^{1}$, Adam Evans ${ }^{2}$, Conor Linehan ${ }^{3}$ \\ ${ }^{1}$ University of Lincoln, Lincoln, UK, kgerling@lincoln.ac.uk \\ ${ }^{2}$ University of Copenhagen, Copenhagen, Denmark, abe@nexs.ku.dk \\ ${ }^{3}$ University College Cork, Cork, Ireland, conor.linehan@ucc.ie
}

\begin{abstract}
Young people using powered wheelchairs have limited access to engaging leisure activities. We address this issue through a two-stage project; 1) the participatory development of a set of wheelchair-controlled, movementbased games (with 9 participants at a school that provides education for young people who have special needs) and 2) three case studies (4 participants) exploring player perspectives on a set of three wheelchair-controlled casual games. Our results show that movement-based playful experiences are engaging for young people using powered wheelchairs. However, the participatory design process and case studies also reveal challenges for game accessibility regarding the integration of movement in games, diversity of abilities among young people using powered wheelchairs, and the representation of disability in games. In our paper, we explore how to address those challenges in the development of accessible, empowering movementbased games, which is crucial to the wider participation of young people using powered wheelchairs in play.
\end{abstract}

\section{Author Keywords}

Games; accessibility; participatory design.

\section{ACM Classification Keywords}

K.8.0 [Personal Computing]: General - Games.

\section{INTRODUCTION}

Young people with disabilities frequently experience barriers when trying to access leisure activities, limiting their opportunities to engage with peers and participate in wider society [31]. Particularly, young people with mobility impairments have limited opportunity to engage in physical play [19]; playful activities which are essential to inclusion, along with the development of physical and social skills [32]. Movement-based games offer a promising opportunity to engage diverse audiences in physically stimulating activities (for example, children with Cerebral Palsy [15],

\footnotetext{
Permission to make digital or hard copies of all or part of this work for personal or classroom use is granted without fee provided that copies are not made or distributed for profit or commercial advantage and that copies bear this notice and the full citation on the first page. Copyrights for components of this work owned by others than ACM must be honored. Abstracting with credit is permitted. To copy otherwise, or republish, to post on servers or to redistribute to lists, requires prior specific permission and/or a fee. Request permissions from Permissions@ acm.org.

CHI'16, May 07-12, 2016, San Jose, CA, USA

(C) 2016 ACM. ISBN 978-1-4503-3362-7/16/05 ..\$15.00

DOI: http://dx.doi.org/10.1145/2858036.2858070.
}

or persons with visual impairments [22]). However, previous research on movement-based games for people using wheelchairs has focused primarily on older players [11], and little research has explored how to design accessible and engaging movement-based experiences for and with young people using wheelchairs. In this context, we are particularly interested in the experiential rather than the exertive nature of movement, focusing on a specific subset of movement-based games that does not lead to exertion, but still enables players to control games through small-scale bodily effort that translates into large-scale wheelchair movement.

In our work, we address this issue through the participatory design of movement-based games for young people who use powered wheelchairs. We establish a structured codesign process with the target audience, and examine how to create games that are engaging for individuals with severe mobility impairment that is sometimes associated with sensory or cognitive impairment. Through this process, we elicit participant values with regard to selfperception, gaming preferences, and movement-based play. Building on these results, we develop three wheelchaircontrolled movement-based games, and present findings from three case studies where young people using powered wheelchairs were invited to engage in play.

Our paper makes the following three main contributions: First, we provide insights into the perspectives that young people with disabilities have on games, providing evidence that game accessibility extends beyond interface design and needs to take into consideration additional aspects such as the representation of disability in games. Second, we provide design considerations for the creation of movement-based games for young people with severe mobility impairment. Third, our case studies reveal challenges and opportunities of movement-based games for young people with mobility impairment, helping inform the work of researchers and designers, and encouraging us to reflect upon common practices in game accessibility.

Understanding how young people with special needs interact with games is an important step in maintaining game accessibility for all audiences. Particularly regarding movement-based games, enabling young people using powered wheelchairs to participate in play offers a first step in increasing their access to physically stimulating leisure activities, which is crucial to wider participation in play. 


\section{RELATED WORK}

This section summarizes findings regarding the involvement of diverse audiences in the design of technology along with previous approaches toward the design of games for players with disabilities.

\section{Involving Diverse User Groups Through Participatory (Game) Design}

Participatory design (PD) - directly working with end-users throughout the development process [23] - is an inclusive approach toward the design of technology.

\section{Diverse Audiences and Participatory Design}

PD offers insights into users' perspectives, and facilitates the design of systems that meets their needs [36], reducing marginalization throughout the research process [30]. This is particularly important when working with audiences with special needs; for example, children or people with disabilities [20].Malinverni et al. [20] outline the opportunity of PD as a means of empowering children with special needs, while Holone and Herstad [16] reflect upon challenges that result from differences in abilities among participants, e.g., the use of proxies in communication.

\section{Participatory Game Design for Players With Special Needs}

A number of projects have applied participatory design in the development of games [18], with some of those exploring participatory design with players with special needs, for example, young people with learning disabilities [1], and children with autism [3]; research by Gerling et al. [13] that compares involving young people using wheelchairs and game design experts shows that both groups produced comparable game concepts, but that young people using wheelchairs had a more realistic perspective on their personal situation. While offering many benefits, a participatory approach also creates challenges, some of which are uniquely associated with game development. For example, Waddington et al. [33] apply PD in the design of therapeutic games for young people with neurological vision impairment. Their results suggest that that while generally effective, PD can expose vulnerability among participants as games are inherently challenging and therefore need to explore players' abilities and disabilities. Likewise, Brederode et al. [5] comment on the risk of PD to expose vulnerability. They explore participatory game design with children with physical disabilities, and highlight that the design process may be physically and cognitively exhausting, and sometimes created stress among participants. Along these lines, Guha et al. [14] underline the importance of a respectful participatory game design process that involves participants in different stages of development based on individual abilities to avoid frustration and disruption.

Generally, these results suggest that PD is an effective way of creating games for young people with special needs, but that careful consideration is required when designing the specific process in terms of setting and methodology, allowing participants to have a positive experience. In our work, we build on these findings to establish a respectful and constructive design process specifically suited for the design and development of movement-based games.

\section{Involving People With Disabilities in Interactive Play}

The involvement of people with disabilities in games and interactive play has been approached from different perspectives, with research focusing on game accessibility, and presenting game development case studies.

\section{Game Accessibility}

Game accessibility is concerned with the adaptation of games to individual needs [34]; frequently, the term is used to refer to ensuring that people with disabilities can gain access to interactive play [26]. Generally, game accessibility research and industry guidelines alike strongly focus on the accessibility of user interfaces and adaptability of game mechanics. For example, in a recent empirical study on game accessibility, Porter and Kientz [26] apply the IGDA Game Accessibility SIG's game accessibility guidelines [17], exploring how game input, output, and mechanics-related aspects such as difficulty levels or settings to reduce speed are integrated in commercially available games. Likewise, Yuan et al. [37] provide a survey exploring game accessibility, offering design strategies around interaction paradigms and the enhancement and adaptation of stimuli provided by games.

\section{Games for Players With Disabilities}

Games for players with disabilities have been explored through numerous case studies, focusing on games for people with sensory, cognitive and physical impairments alike. For example, Morelli and Folmer [22] designed a range of audio-guided exergames for players who are blind, Rector et al. [28] created eyes-free yoga, an exergames for people with visual impairments, and Bhattacharya et al. [2] explored interactive play to engage children with autism. Addressing the design of games for players with physical disabilities, a number of projects have explored the potential of movement-based games, often focusing on their potential to support therapy and rehabilitation and to encourage players to be more physically active (e.g., playful therapeutic environments for children with physical disabilities [27]). In this context, findings from the design of exergames for children with Cerebral Palsy by Hernandez et al. [15] show that players have a preference for fast-paced action games rather than engaging in slowpaced experiences, suggesting a need to re-think common game accessibility recommendations.

\section{Wheelchair-controlled Movement-based Games}

Different technical approaches have been made exploring the development of wheelchair-controlled movement-based games. In terms of stationary systems, O'Connor et al. [24] present GAME ${ }^{\text {Wheels }}$, a custom-built mechanical system that wheelchairs can be mounted on, and that was designed to translate wheelchair propulsion into game input. Furthermore, Cuzzort and Starner [6] developed AstroWheelie, an arcade game that leverages accelerometer 
information to track wheelchair movement, and Gerling et al. [11] created KINECT ${ }^{\text {Wheels }}$, a vision-based wheelchair tracking system for game input that records basic wheelchair movement (turning to sides, and moving back and forth). Exploring GPS tracking, Edey et al. [7] offer insights into mixed reality gaming for persons using powered wheelchairs. Additionally, previous work has provided recommendations for wheelchair-controlled movement-based games [11], focusing on factors such as appropriate movement patterns, and the impact of wheelchair models and propelling techniques on gameplay.

Generally, research in the area of wheelchair-controlled movement-based games focuses narrowly on improving the accessibility of interface design and game mechanics, leaving many questions around game design and player preferences unanswered. However, none of the previous projects directly involved users in the design process, and to provide truly accessible and empowering experiences, a better understanding of player perspectives on movementbased play is necessary. In our work, we aim to address this issue by exploring the participatory design of wheelchaircontrolled movement-based games for players with mobility impairment with a focus on player preferences, and perspectives on the value of movement-based play.

\section{DESIGNING MOVEMENT-BASED GAMES WITH YOUNG PEOPLE USING POWERED WHEELCHAIRS}

At the heart of our research is a co-design process that involved young people using powered wheelchairs in the design of movement-based games that can be controlled using wheelchair input.

\section{Research Site and Participants}

We worked with St. Francis School in Lincoln, UK, a school that provides education for young people between the ages of three and nineteen who have special needs. St. Francis School primarily focuses on students with physical disabilities, but also caters to individuals who have associated conditions, for example, sensory or cognitive impairment. Many of the students use mobility aids, and a large share of young people use powered wheelchairs to navigate their environment. Throughout the day, the school offers a range of activities including sports, arts and crafts, activities that are tailored to students with complex needs (e.g., sensory experiences), and excursions; however, extracurricular activities are often challenging given the range of abilities and needs among students.

Nine young people (three female, age range 13 to 22) took part in the design sessions. All participants experienced severe mobility impairment and used powered wheelchairs, six participants also had sensory or cognitive impairments. The majority of participants had been living with the mobility impairment since birth (e.g., as the result of a progressive neurodegenerative disease such as Cerebral Palsy), two participants had to adapt to the use of a wheelchair as the result of an accident. All participants had played games before, and most participants were familiar with the concept of movement-based gaming technology (e.g., Nintendo Wii Remotes and Microsoft Kinect) and were aware of movement-based games such as Nintendo Wii Sports Bowling. However, none of the participants had been able to engage with such games due to access-related barriers. Regarding opportunities for physically active play, St. Francis School does provide opportunities for PA for all their students, but only one participant reported playing wheelchair sports as a hobby, and another participant reported going on (assisted) bike rides with a family member, while many other participants reported sedentary hobbies such as reading, spending time on social networks, listening to music, or watching TV.

The research was approved by the University of Lincoln College of Science ethics board, and granted operational approval by St. Francis School. Written consent was obtained from parents, and we followed an assent protocol in which the project was explained to the participants. During design sessions researchers were accompanied by school staff, who also supported participants with difficulties expressing themselves.

\section{Participatory Game Design Sessions}

Guided by our overall research question - exploring the value of movement-based play for young people using powered wheelchairs - we created a set of four participatory design sessions each designed to explore a specific aspect of movement-based games while also touching upon disability and mobility aids. From each of the sessions, we hoped to learn about a specific aspect of game design, for example, exploring participants' gaming interests, self-perception and how that would affect playful experiences, along with suitable input methods. Over the course of four months, we hosted a total of nine sessions (each theme was repeated for two separate groups, attended by an average of four participants, with one individual arrangement) in which we invited participants to collaborate with us on the creation of game concepts suitable for wheelchair input. All sessions were audiorecorded and transcribed. Where appropriate, interview data was analyzed using Deductive Thematic Analysis [8] following a protocol proposed by Braun and Clarke [4]; transcripts were thoroughly read by one researcher and coded following the research questions behind each session.

\section{Session 1: Introduction and Brainstorming}

This session was designed to explore design requirements and identify desirable game themes. To this end, we asked guiding questions making enquiries into participants' backgrounds and gaming preferences, encouraging them to broadly explore game themes of interest.

Three main themes emerged throughout analysis: player abilities, contextual factors, and gaming preferences, all of which influenced the kinds of games participants engaged with and were interested in. The first theme, player abilities, touched upon the impact of individual abilities on gaming habits. When discussing previous gaming experiences, 
accessibility was frequently touched upon. For example, one participant expressed that she "would like to [play Xbox and Wii games] but I can't use my hand" and that "it takes a lot of strength" (P8), suggesting that physical challenges influence the range of games available, making her opt for tablet-based games that can be controlled using a head switch instead. Along these lines, one participant with cognitive impairment expressed frustration with commercially available games, stating that he "can't do it [play console games]" (P5); in this context, staff also pointed out difficulties finding suitable games for young people interested in sensory experiences. Additionally, the second theme revealed the impact that contextual factors had on their choice of games, for example, the impact of parents, "I don't tend to play a lot of first-person shooters, because I find them... my parents find them really hard to control, or um, they're very gory", and time available to play, e.g., "[...] sometimes at school if I can find games that is not blocked or whatever or if I've got a spare 15 or 20 minutes I'll y'know sometimes play a bit of that and it lets me sort of take a break" (P1). In this context, many participants commented that they played casual games (e.g., social network games) as these would allow them to fit short chunks of play into their day.

The third theme, gaming preferences, encompassed game themes, features, and desirable player experiences. While playing a range of games on various platforms (from social games to platformers such as Super Mario, and first-person shooting games such as Call of Duty) in their spare time, all participants were interested in sports games, suggesting that they enjoyed fast-paced gaming experiences, which is in line with previous findings on accessible game design by Hernandez et al. [15]. Particularly, participants suggested designing skiing, rock climbing, boxing, and driving (i.e. racing) games. Participants suggested that these types of games would give people using wheelchairs an opportunity to experience activities that mostly remain inaccessible in their daily lives, for example, with one participant reflecting about his peers (but interestingly not himself), stating that "it would be quite nice for them to have something like... that they wouldn't get a chance to have a go, some kind of reality thing they that could have a go at they wouldn't have a chance to normally" (P1). When enquiring about specific elements that make games enjoyable, participants reported competition along with the opportunity to experience competence as one of the most engaging factors, for example, when reflecting upon past experiences: "I was playing Call of Duty and I beat a... I beat a guy that was like prestige four and I was pretty new. So I was pretty proud of that" (P9). Yet, participants were also mindful of negative aspects of competition, suggesting that "we don't want it to be upsetting to be... too upsetting when one child beats another child but, um, we don't want it to be too boring for them either" (P1), which seems particularly important considering the range of physical and cognitive abilities among participants.

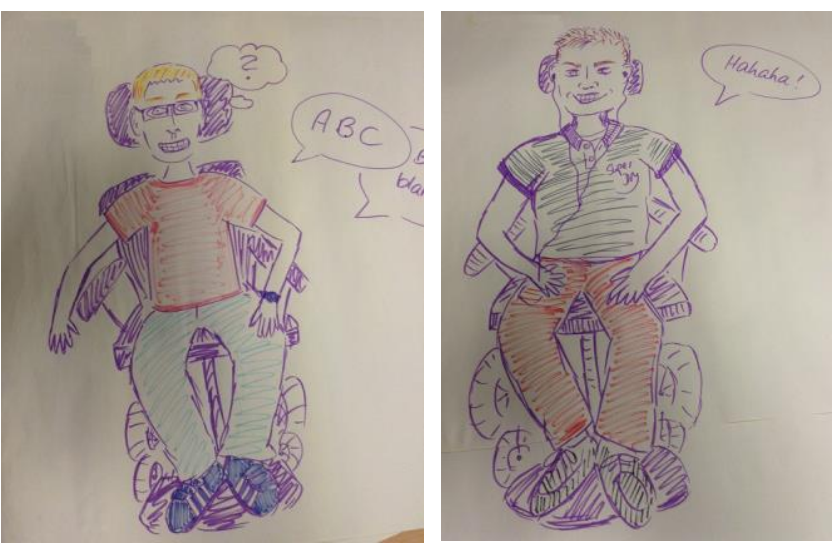

Figure 1. Examples of drawings that were produced together with study participants in Session 2.

\section{Session 2: Self and Player Representation}

In the second session, we applied techniques derived from visual sociology [25], and produced drawings of participants based on their descriptions to derive insights into how they viewed themselves, helping us inform player representation within the games, and assess the suitability of wheelchair-based interaction for this audience. Additionally, we carried out semi-structured group interviews exploring participants' self-perception and thoughts on in-game representation, e.g., whether avatars should reflect a mobility impairment to explore questions around the potential benefits of avatar customization for player experience [9].

When asked to instruct drawings, most participants begun to describe aspects of their personality (e.g., having a sense of humour, or being talkative), rather than outer appearance. Considering wheelchairs, six out of eight participants considered their wheelchair an important part of themselves, asking for it to be included in the pictures, while the other two preferred being depicted as nondisabled persons, one participant specifically asking to be depicted playing rugby. Themes that emerged from interviews discussing in-game representation focused on avatar appearance, and more prominently, in-game abilities. While visual features were mostly discussed through examples of other games, reflection upon desirable abilities strongly focused on physical aspects, e.g., strength and speed. Participants linked in-game abilities with their own situation, for example, one participant pointed out that their preferred avatar would be "something that was sort of something that was more like me", but that it would give them "characteristics that weren't like me. Like maximum strength, accuracy, power all that sort of stuff" (P1).

When specifically asked about an avatar that would have a mobility disability, participants were apprehensive, with only two out of eight participants asking to be represented by an avatar that uses a wheelchair, despite earlier responses suggesting that they considered their wheelchairs an important part of themselves. In this context, one participant asked about in-game limitations, wondering 
whether it would be "difficult because you wouldn't have the same range of movement, so it would effectively be harder but in other ways it's kind of, It's kind of very... like at least if I was able bodied you wouldn't have to worry about not having the same range of movement" (P1). Another participant commented that he simply wasn't used to seeing disability in games, stating that his "natural preference would probably be able-bodied but that's because obviously there's not to my knowledge, there's no games I've ever come across where there's been a guy in like a wheelchair or any sort of", and highlighting that he was "naturally used to playing as an able bodied person" (P9). This shows that perceptions of disability in games were shaped by their daily encounters with accessibility barriers as well as previous gaming experience, suggesting that challenges and opportunities resulting from the representation of disability in games need to be carefully researched to create positive, empowering experiences.

\section{Session 3: Interaction Design}

This session was driven by technology and focused on perceptions of different game input devices, including hands-on testing of input devices including an assistive joystick, a traditional game pad, and the Microsoft Kinect. Furthermore, this session explored participants' relationships with mobility aids (i.e., different kinds of wheelchairs), and their potential for game input.

Main themes that emerged during analysis were independence and accessibility. When discussing perspectives on mobility aids, participants had very strong feelings about manual wheelchairs, with one participant spontaneously exclaiming that he "hates it" (P5). Following up on this comment, other participants explained that manual wheelchairs were pushchairs that could only be used with the help of others, whereas powered wheelchairs gave them the freedom to independently navigate their environment, with one participant commenting that she "saw herself" when looking at pictures of powered wheelchairs (P8).

Likewise, participant responses to devices for computer input were similar, focusing on enabling aspects. While two participants commented that they had previously used gamepads, participants with fine motor impairments commented that they did associate gamepads with playful experiences, but also accessibility barriers. This ambivalence reflected some of the findings from the first session regarding accessibility issues when using traditional game controls. Most importantly, many participants expressed preference for input that either leveraged movement of their wheelchair, or built on similar technologies than those they used to control their wheelchairs (e.g., switches or gaze input). This would enable them to control games in a familiar way, rather than learning and adapting traditional input devices, with one participant stating that they "already are experts at driving wheelchairs, so why can't we use this to play" (P1).

\section{Design Implications Resulting From Sessions 1-3}

Building on the results of the first three design sessions, we identified the following implications for the design of games for young people using powered wheelchairs:

(1) Genres and themes: Games should be casual in nature, allowing for short chunks of play to accommodate the players' environment, and provide enjoyable experiences for players who may experience fatigue after short bouts of play. Sports or sports-like experiences are game themes likely to appeal to broad groups of players; generally, players expressed preference for themes that relate to realworld experiences.

(2) Game elements and features: Game elements enabling competition between players were a common theme that emerged from the design sessions. However, given the heterogeneity of cognitive and physical abilities within the target audience, designers are challenged to integrate adequate balancing strategies if player performance is compared to ensure positive, encouraging competition. With regards to representation of disability in games, it is important to integrate such elements (e.g., avatars that have a disability) in a way that empowers players to have positive in-game experiences, rather than limiting their abilities within the virtual world.

(3) Game controls: Game controls need to accommodate an extremely wide range of abilities among players, with some being able to use traditional game controllers, and other players having to rely on assistive technology as for example head switches or gaze-based interaction. To this end, wheelchair-controlled play offers an interesting design opportunity as many young people using powered wheelchairs will be able to navigate their wheelchair independently, and will not require additional support.

Building on these implications, we worked with a range of game themes proposed by the participants, and created high-level game concepts. We then returned to St. Francis School to discuss and further develop these concepts in a final design session.

\section{Session 4: Game Concepts: Mechanics and Refinement}

The last session was designed to tie together results from the previous sessions, offering opportunity for participants to further explore themes, ideas for player representation, and perspectives on game input. We guided this process through an overview of suggestions that came out of the first three sessions, allowing participants to reflect upon and refine their ideas.

To this end, we prepared six game concepts that were derived from the initial interviews: A rock climbing game in which players can move up to the top of the mountain by guiding a climber to pick a safe route, a boxing game that 

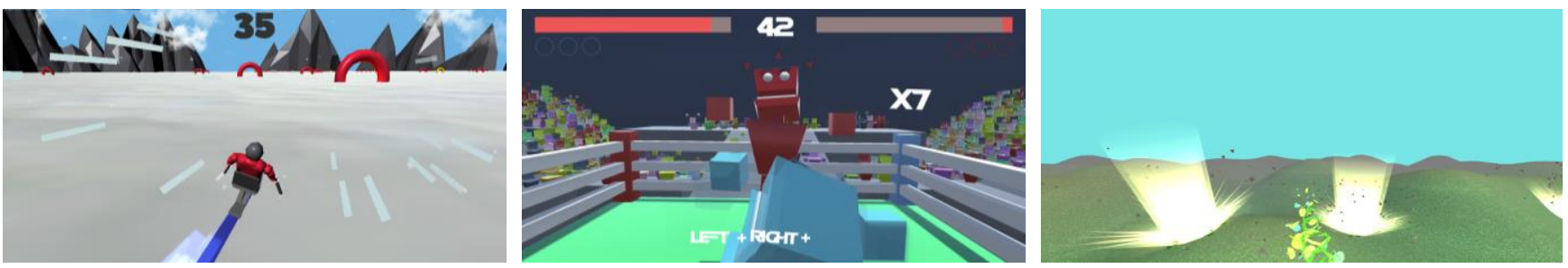

Figure 2. Gameplay in Speed Slope (left), Rumble Robots 3D (middle), and Rainbow Journey (right).

allows players to compete against an opponent carrying out punches and blocking attacks, a more accessible, adaptable version of the arcade game PacMan, a downhill skiing game where player input translates into turns, a sensory experience that does not set goals but provides rich visual and auditory feedback, and a bumper car racing game. The concepts were presented to and discussed with the participants, reflecting upon their personal opinions along with the potential of games to appeal to other players, and suitability of mapping wheelchair input onto in-game actions. Based on participant feedback, three game concepts were selected for further development.

\section{Overview of Game Concepts}

Based on the outcome of the participatory game design sessions, three concepts were chosen for implementation into playable games: downhill skiing, boxing, and a sensory experience. We decided to implement wheelchair-based control schemes (moving back, forth, and turning to the sides to make input) as these would keep the games accessible for a broad range of players regardless of individual differences in fine and gross motor skills, and would allow us to further explore the value of movementbased input for young people using powered wheelchairs. All games were implemented using Unity 5.0 and an extended version of KINECT ${ }^{\text {Wheels }}$ [11]

\section{Game 1: Speed Slope}

Speed Slope (Figure 2, left) is a downhill skiing game in which the player uses an assistive skiing device, and is challenged to reach the foot of the mountain while trying to maximize their score. This game was selected as many participants expressed interest in being able to experience an activity that would often remain inaccessible, giving them a playful insight into the sport.

Gameplay. The avatar automatically speeds up while the player can decide to make left and right turns. Momentum slowly builds up over time, increasing player speed. To add an element of challenge, the slope is populated with arches that increase the player's speed, allowing them to aim for higher scores by steering through the rings. Once the time limit is reached, the player's score is displayed based on distanced traveled down the mountain.

Controls. To start the game, players are asked to quickly wheel back and forth. To control the direction of the avatar, turning the wheelchair left or right directly translates into change of direction within the game.

\section{Game 2: Rumble Robots 3D}

Rumble Robots $3 D$ (Figure 2, middle) is a fighting game in which the player controls a robot boxer, and must defeat the opposing robot. This game was chosen as participants expressed an interest in action-based games that would enable them to control strong characters; we decided to set the game in a sci-fi environment to maintain suitability for players of all ages.

Gameplay. The gameplay of Rumble Robots is broken down into round-based boxing; the main objective of the player is to win three rounds. Each round lasts 60 seconds or until one of the robots' health is fully depleted. The player robot is able to swing punches toward the opposing robot and block punches from the opposing robot. These mechanics cost stamina, which is a player based resource that regenerates during periods of inactivity, challenging the player to balance offense and defense. Series of punches (e.g., left, right, left) unlock special combo moves that deal more damage combined with special animations.

Controls. The robots' fists are controlled in real time by wheelchair turns; for example as the player turns to the left the left fist will start moving backwards showing it is preparing to punch, then when the player turns back to the right the left fist is thrown forward with the speed that they turned. Additionally, moving the wheelchair back triggers block mode to avoid the opponent's attacks.

\section{Game 3: Rainbow Journey}

Rainbow Journey (Figure 2, right) is an interactive experience that was designed to engage players through graphics and sound, but does not include objectives or goals. Given its experiential nature, it is particularly suited for persons with an interest in sensory environments, and players with cognitive impairments that would make it difficult to engage in goal-based play requiring clear understanding of game rules.

Gameplay. The player assumes control of an avatar consisting of leaves and flowers that floats around a procedurally generated world by its own volition. Although not required to be able to experience Rainbow Journey, players can change the colour of the game world, and spawn golden globes which fly towards the ground and on colliding with terrain explode into pillars of light and spawn flowers where the globe landed, creating rich visual feedback if the player interacts change to ensure feedback. 
Controls. Rainbow Journey interacts with the wheelchair in several ways to allow the player to influence the visual state of the game world. Moving backwards or forwards changes the color of the world; to ensure players remain centered, forward movement needs to be followed by backing up, and vice versa. The other opportunity for player input is spawning orbs, which is done by turning to the left and right. If players do not make any input, the avatar will keep floating through the game world.

\section{CASE STUDIES: EXPLORING THE VALUE OF MOVEMENT-BASED GAMES FOR YOUNG PEOPLE USING POWERED WHEELCHAIRS}

We carried out qualitative enquiries into the experience that young people using powered wheelchairs had when engaging with the games to explore the value of movementbased play for this audience. Furthermore, we contribute insights into the experience that players with a range of abilities had when engaging with our games.

\section{Research Questions}

We were interested in two main research questions around the games that we built, further investigating the aspect of movement and player abilities: (1) Are the games accessible and engaging for young people using powered wheelchairs, and is wheelchair movement a suitable input modality? (2) How do individual differences (e.g., gaming experience, cognitive abilities) between players influence the interaction and experience with the games? Based on these questions, we hope to better understand the player experience that young people using powered wheelchairs have, allowing us to elicit the value that movement-based play may have for this audience.

\section{Study Design and Data Analysis}

We returned to St. Francis School, and participants were recruited through staff. Again, written consent was obtained from parents (except for two participants who had taken part in the first stage of the project where parents had already consented to their participation), and we followed an assent protocol in which this stage of the project was explained to the participants. These sessions were facilitated by a team of two researchers, who were accompanied by school staff.

All case studies followed the same pattern where we gave participants a brief introduction to the games and collected demographic information, followed by independent playing time. During this period, participants received no instruction other than engaging with the games to their liking. Researchers were available to explain control schemes of the games, and to provide technical assistance such as re-calibrating the tracking system. This phase lasted between 15 and 45 minutes. Afterwards, we followed up with participants in a semi-structured interview that explored their experience with the games (e.g., themes and controls) and their thoughts on movement-based play.

All sessions were audio-recorded and transcribed. Additionally, one researcher logged observations to allow us to follow an adapted version of Thick Description [10] where we collated observations and audio records into one document to facilitate a deeper understanding of how participants engaged with the games, and enable us to relate players' experiences to contextual factors. Data analysis was guided by the research questions and carried out using Thematic Analysis as outlined by Braun and Clarke [4]; transcripts were thoroughly read by one researcher and coded accordingly.

\section{Results}

In this section, we describe the case studies. For each one, we describe the participants' individual background(s), the context in which they played the games, followed by an overview of main themes that emerged from the analysis of gaming sessions along with interview results.

\section{Case Study 1: Daniel}

The first case study outlines the experience of Daniel, who enjoys games and has been using a powered wheelchair through gaze control for a number of years.

Participant's background. Daniel is 18 years old, and generally interested in video games, with Skylanders, an action-based platformer for younger players being his favourite game. Daniel has been using a wheelchair for five years, following a severe traffic accident which resulted in a spinal cord injury that left him paralyzed from the neck down. Daniel has no control over his arms and hands, and experiences difficulties speaking. To communicate, he uses a gaze-controlled communications tool which combines an eye tracker with a tablet and sound output; to control his wheelchair, he uses a head switch. However, technical limitations of this solution do not allow him to speak and steer the wheelchair at the same time, and he requires assistance to switch from communications to wheelchair control mode and vice versa.

Context of play. The session took place in a medium-sized room at St. Francis School that offers some equipment for sensory stimulation, but was mostly empty except for two chairs and two small tables. Additionally, the room featured a smartboard that we used to display the games. The session lasted about 45 minutes, included all three games, and was accompanied by two researchers, and two members of staff.

Player experience. There were two main themes that emerged throughout analysis, and are of interest with regards to understanding the player experience of Daniel. The first theme is concerned with the accessibility of wheelchair game controls. While all games were generally accessible, the head switch that Daniel used to control his wheelchair introduced small amounts of delay that made it more challenging for him to reach in-game goals. While the effect was negligible in Rainbow Journey, and relatively small in Rumble Robots 3D, Daniel's performance in Speed Slope was heavily affected by his control system as the game required higher levels of wheelchair control, suggesting that such games need to offer flexibility not just 
in terms of adapting in-game challenge to player ability, but also to their equipment. In this context, the post-play interview showed that this higher level of challenge did not affect Daniel's perception of the games, pointing out that he "liked them all". The second theme that emerged was the expression of emotion through movement. On several occasions, Daniel showed excitement about in-game events, e.g., smiling when hitting his opponent while boxing or planting a flower in Rainbow Journey. More notably, he also moved his wheelchair to express enjoyment, quickly moving back and forth or turning to the sides. As our game was designed to process these movements for game input, this sometimes resulted in erroneous input, and also affected alignment with the Kinect sensor as Daniel moved without directing his attention to the projection area. This might be a result of Daniel being unable to communicate verbally while moving his wheelchair, suggesting that future game designs should consider enabling players with (situational) verbal impairment to express emotions through wheelchair movement.

\section{Case Study 2: Mark}

The second case study reflects upon the experience of Mark, a young man with a cognitive impairment.

Participant's background. Mark is 17 years old, and has been using wheelchairs all of his life. He first started using powered wheelchairs at the age of 8 , and is now often transitioning between powered and manual wheelchairs. Mark occasionally plays video games on the Nintendo Wii console, and has a keen interest in music. His cognitive impairment influences his ability to communicate, and also makes it difficult to follow complex games.

Context of play. The session took place on the same afternoon as the previous one, was held in the same room, and lasted about 30 minutes. During that time, Mark chose to play Speed Slope, Rumble Robots 3D, and the sensory experience Rainbow Journey. The session was accompanied by two researchers, and two members of staff. Throughout play, Mark was assisted by a member of staff to control his wheelchair, helping him follow the input movements required by the games. Because Mark has difficulties expressing himself through speech or writing, he was supported by staff making suggestions about his perspective, and then nodding or shaking his head to express agreement or disagreement.

Player experience. The dominating theme that emerged from analysis was support required to play, both in terms of game controls as well as understanding conceptual aspects, for example, game rules. While Mark generally seemed to respond to the games and there was some evidence of enjoyment (e.g., pointing at the projection area, laughing in response to in-game events, and moving the wheelchair similar to observations made in the previous case study), he required assistance throughout the session to be able to engage with the games. Regarding game controls, he was given an introduction to each of the games, but did not follow the pattern throughout play, for example, moving his upper body instead of the wheelchair, or engaging in wide movements that were not supported by our tracking system. To this end, we offered additional guidance on how to best move the wheelchair to play the games, and staff supported Mark when navigating his wheelchair, e.g., by helping him re-align himself with the projection area. Additionally, there were some instances where Mark experienced difficulties trying to maintain focus and understanding the rules of the games. For example, when Mark was asked whether he wanted to try the robot boxing game, he formed fists and carried out boxing movements, focusing on his carer, who tried to draw his attention to the game: "Yeah? Game on. Mate, you're not boxing me, you're boxing the game. It's on the screen. " Likewise, when playing Rainbow Journey, he visibly enjoyed graphical effects (e.g., growing flowers) in the game, but did not seek to carry out movements that would trigger these in-game events, suggesting that he was engrossed in the visuals without understanding the connection with player input.

\section{Case Study 3: Samuel and Matt}

The third case study reflects upon the experience that Samuel and Matt had when engaging with the games, two participants with a strong interest in games, and previous gaming experience spanning casual games on social networks to triple-A console titles.

Participants' background. Samuel (age 16) and Matt (age 17) are friends who were part of the participatory design process leading to the game concepts presented in this paper, and decided to attend the gaming sessions together. Both have a keen interest in video games, Samuel preferring sports- and racing games such as Forza on the Xbox One, but also having an interest in casual games, and Matt mostly playing FPS games, with Call of Duty and the Grand Theft Auto series being his favourite games. Samuel and Matt are long-term users of powered wheelchairs as a result of neurodegenerative diseases such as Cerebral Palsy; both of them have control over their upper limbs, and use joysticks for wheelchair control.

Context of play. Samuel and Matt played the games on two afternoons over the course of two weeks, the first lasting about 1.5 hours, and the second lasting an hour. Both sessions were hosted at St. Francis School, the first in a quiet, smaller room dedicated to recreational activities within the residential unit, and the second in an IT classroom that offered more space to facilitate switching between players. Throughout the gaming sessions, Samuel and Matt took turns testing the games, starting out with Speed Slope and Rumble Robots 3D, and also trying out the sensory experience Rainbow Journey in the final session. All games were played on a laptop with a 17" widescreen display, and we ensured that players were able to follow gameplay even when seated at a distance due to wheelchair movement. While playing the games, the atmosphere was open, with both participants occasionally teasing each other 
and taking an interest in their scores. Both sessions were accompanied by two researchers, and a member of staff.

Player experience. There were three main themes that emerged throughout analysis, the accessibility and appeal of game wheelchair controls, competition between players, and the representation of disability in games.

Observations, along with participant comments showed that accessibility and appeal of wheelchair game controls were good, with both Samuel and Matt quickly picking up the control schemes, and pointing out that they enjoyed all three games. Feedback on the idea of using wheelchair movement for game input was very positive, with Matt commenting that it actually improved the accessibility of games for Samuel, who struggles with traditional game controls: "You've normally got a controller in your hand as well. Which [using a wheelchair for input] I suppose makes it easier for you as you find it harder with a controller. Whereas with steering you're actually sort of semi good at. [jokingly]" There were some minor issues regarding calibration, i.e., the location the player needed to be in to make input, with Samuel commenting that "I never know how far back you gotta be for it.", suggesting that players need more guidance in terms of wheelchair alignment. With regards to the themes and general design of the games, Samuel stated that "I think you've got the ideas of the games pretty much down to a T from what we discussed", and Matt agreed that he thought that "they were really, really good", suggesting that the games met their expectations following the participatory design sessions.

The second theme that emerged throughout analysis was competition. Initially, Samuel and Matt were keenly interested in their scores, joking about Matt's competitive personality, and discussing whether they had accomplished a better result than their peer. However, observations throughout the sessions revealed that Matt consistently scored higher than Samuel as a result of better wheelchair control. Sensing Samuel's frustration about this, Matt ceased to comment on scores and supported Samuel with tips on how to do better instead, mindfully managing a situation that could have exposed vulnerability. Specifically commenting on the role of competition in games, Samuel pointed out that "It's cool in the sense that you've got the two sort of competitive games but then you've just got that game where you don't you know you can just relax and watch what happens so I think it's quite a good balance in that sense.", and both participants agreed that games without a predefined goal such as Rainbow Journey might be well suited for players with a wide range of abilities.

Finally, the representation of disability was extensively discussed by Samuel and Matt, who immediately noticed the inclusion of an assistive skiing device in Speed Slope rather than showing a non-disabled skier. Both participants expressed excitement about the design decision, pointing out that "It makes me feel more like it's catered for us rather than just sort of afterthought." (Samuel), and that
"[...] it means something to us because it's relevant to our situations like its kind of cool to be able to see that." (Matt). Additionally, there was reflection on the implications of in-game representation, suggesting that it offered of painting a more comprehensive picture of people with disabilities, with Matt pointing out that it "[...] proves that being in a chair isn't you know, you." In response, Samuel reflected on their abilities, pointing out that "There's no boundaries!', and that such games do not only speak to, but possibly also represent people with disabilities, "It's kind of cool because you look at it and go well actually this game was designed for people like us and that shows people like us."

\section{Main Findings from the Case Studies}

Our case studies offer a number of insights into movementbased play for young people using powered wheelchairs. Across all case studies, initial calibration of player location and alignment throughout play was an area that created accessibility issues, and needs to be improved on in the future. Furthermore, all case studies highlighted the impact of cognitive and physical player abilities on player performance, suggesting that even small differences in wheelchair control can translate into substantial score differences. Additionally, there were three interesting individual findings in our case studies. First, our results suggest that the representation of disability in games can improve the player experience of young people using wheelchairs. Second, there was some evidence that complex needs have a profound impact on player access to games, and third, that players not only use their wheelchair to navigate their environment, but use wheelchair movement to express emotion, which has implications for the design of wheelchair-controlled games.

\section{DISCUSSION}

In our work, we focus on the participatory design of wheelchair-controlled movement-based games for young people using powered wheelchairs. We provide design recommendations based on a participatory design process, we present three fully playable games that were developed based on these considerations, and we explore the experience they provide for young people using powered wheelchairs through case studies. Our findings suggest that participatory design has potential to facilitate the development of engaging games, while giving the audience a voice throughout development. Follow-up case studies showed that resulting games were engaging for young people using powered wheelchairs, but that there are challenges in movement-based play and game accessibility that need to be addressed in the future. In the following sections, we discuss the value of movement-based play for persons with mobility impairment, and we reflect upon implications of our work for game accessibility research.

\section{Exploring the Value of Wheelchair-Controlled Movement-Based Play}

Traditionally, research exploring movement-based games has strongly focused on benefits that result from the effects 
that physical activity has on players' bodies. We see two main benefits that wheelchair-controlled movement-based play may have regardless of the levels of exertion that such games provide, potentially providing valuable experiences for people with severe mobility impairment. First, our results show that integrating powered wheelchairs in game control offers the opportunity of enabling players to control games through a device that they are already familiar with, and can competently use. Reflecting on the importance of ability-based design [35], this suggests that wheelchaircontrolled games build on the player's expertise in wheelchair control, rather than asking them to use traditional game controls, which are often inaccessible, or learn how to use assistive game input devices that will come with a learning curve. Second, movement-based play offers the opportunity of diversifying leisure activities available to young people using powered wheelchairs, giving them an opportunity to explore their physical body in a playful context, possibly giving them access to some of the psychological benefits of physical play [31].

\section{Reflections on Game Accessibility}

In this section, we reflect upon the implications of our findings for game accessibility, particularly exploring the role of disability in games, and whether 'one size fits all' game design is appropriate for all player groups.

\section{Player Perspectives on Disability in Games}

While the representation of disability has broadly been discussed in film and literature [21], game accessibility has traditionally focused on the accessibility of games in terms of interaction paradigms and game mechanics. Our work reveals that a broader approach may be necessary, taking into consideration the integration of disability in games, while being mindful of the implications of disability for participation in society. Our findings suggest that despite considering their assistive device an important part of themselves, young people with disabilities are not familiar with examples of disability in games, and were apprehensive about its inclusion as they were concerned about limitations that might be introduced into play. However, opinions were favourable when considering the integration of positive images of disability in games, and feedback suggested that representing disability in games in a positive context offers the opportunity of taking a major step towards allowing players with disabilities to see themselves in games, possibly facilitating a deeper, more personal experience that could have implications for player experience [9]. Additionally, this may also encourage nondisabled players to reflect upon their perspectives on disability, similar to effects seen in film [29].

\section{Designing for Diversity - Does One Game Fit All?}

Game accessibility is often addressed through guidelines (e.g., [37]) and the design of games addressing challenges that player groups with specific impairments may experience (e.g., [11, 15]). Our findings suggest that we need to adopt a more differentiated view. While smaller performance differences could be offset through balancing strategies [12], we also observed that differences in cognitive abilities influenced players' abilities of understanding game rules and subsequently achieving ingame goals. Although we pre-empted some issues through the breadth of games we offered, our results raise the question whether any game can be made accessible for all players, and what alternative approaches to accessible game design could look like. We believe that sandbox-style games that encourage players to set their own goals might offer a design opportunity - while designers would still have to create accessible game interfaces, this would empower players to adapt games to their needs, especially if one game has to accommodate diverse audiences.

\section{LIMITATIONS AND FUTURE WORK}

There are a number of limitations that need to be considered in the context of this project. Most importantly, the findings we present here are the result of a single project with a small number of participants, and need to be viewed in this light. Additionally, gaming sessions only looked at shortterm player experience and participants were exclusively male; future work should explore the thoughts that female players have on wheelchair-controlled games. Nevertheless, we believe that our work provides valuable insights into the perspectives that young people using powered wheelchairs have on games. Specifically regarding the importance of representation of disability in games, we believe that it would be important to follow up on this topic on a broader scale, investigating perspectives of disabled and nondisabled players, and how games could integrate disability in an empowering way. Likewise, future work should investigate the design of games for players with different cognitive abilities, exploring the idea of sandbox-style play to accommodate a range of player abilities and interests.

\section{CONCLUSION}

Enabling young people with disabilities to participate in society is an important step towards increased well-being and quality of life. Physically engaging playful activities are an integral part of connecting young people with their peers, however, young people with mobility disabilities often struggle to gain access to such activities. Our work suggests that the participatory development of movementbased games has potential to create engaging playful experiences with a physical dimension. However, findings also suggest that we need to move beyond common approaches to game accessibility, not only creating accessible game interfaces and mechanics, but also developing inclusive game content that appeals to players with disabilities: We need to ensure that games reflect how players - including young people with disabilities - view themselves, and enable them to become who they strive to be through engaging and empowering playful experiences.

\section{ACKNOWLEDGMENTS}

We would like to thank all study participants and St. Francis School staff for supporting our research. The project was funded by the University of Lincoln Research Investment Fund. 


\section{REFERENCES}

1. Lisa Anthony, Sapna Prasad, Amy Hurst, and Ravi Kuber. 2012. A Participatory Design Workshop on Accessible Apps and Games with Students with Learning Differences. In: Proceedings of the $14^{\text {th }}$ International ACM SIGACCESS Conference on Computers and Accessibility (ASSETS '12), 253-254. http://dx.doi.org/10.1145/2384916.2384979

2. Arpita Bhattacharya, Mirko Gelsomini, Patricia PérezFuster, Gregory D. Abowd, and Agata Rozga. 2015. Designing Motion-Based Activities to Engage Students with Autism in Classroom Settings. In: Proceedings of the $14^{\text {th }}$ International Conference on Interaction Design and Children (IDC '15), 69-78. http://dx.doi.org/10.1145/2771839.2771847

3. Laura Benton, Hilary Johnson, Emma Ashwin, Mark Brosnan, and Beate Grawemeyer. 2012. Developing IDEAS: Supporting children with autism within a participatory design team. In: Proceedings of the SIGCHI Conference on Human Factors in Computing Systems (CHI '12), 2599-2608. http://dx.doi.org/10.1145/2207676.2208650

4. Virginia Braun and Victoria Clarke. 2006. Using Thematic Analysis in Psychology. Qualitative Research in Psychology 3, 2 (July 2008), 77-101. http://dx.doi.org/10.1191/1478088706qp063oa

5. Bas Brederode, Panos Markopoulos, Mathieu Gielen, Arnold Vermeeren, and Huib de Ridder. 2005. pOwerball: The design of a novel mixed-reality game for children with mixed abilities. In: Proceedings of the 2005 Conference on Interaction Design and Children (IDC '05), 32-39. http://dx.doi.org/10.1145/1109540.1109545

6. Stephen Cuzzort and Thad Starner. 2008. AstroWheelie: A wheelchair based exercise game. In: Proceedings of the $200812^{\text {th }}$ IEEE International Symposium on Wearable Computers (ISWC '08), 113114. http://dx.doi.org/10.1109/ISWC.2008.4911599

7. Jamal K. Edey, Katie Seaborn, Carmen Branje, Deborah I. Fels. 2014. Powered to play: A mixed reality game for people driving powered chairs. In: Proceedings of 2014 IEEE Games Media Entertainment (GEM), 1-8. http://dx.doi.org/10.1109/GEM.2014.7048101

8. Jennifer Fereday and Eimear Muir-Cochrane. 2006. Demonstrating rigor using thematic analysis: A hybrid approach of inductive and deductive coding and theme development. International Journal of Qualitative Methods 5, 1 (2006), 80-92.

9. Waddell T. Franklin, Sundar S. Shyam, and Auriemma Joshua. 2015. Can Customizing an Avatar Motivate Exercise Intentions and Health Behaviors Among Those with Low Health Ideals? Cyberpsychology,
Behavior, and Social Networking 18, 11 (2015), 687690.

10. Clifford Geertz. 1973. Thick Description: Toward an InterpretiveTheory of Culture. The Interpretationof Cultures: Selected Essays. Basic Books: New York.

11. Kathrin M. Gerling, Regan L. Mandryk, Matthew Miller, Michael R. Kalyn, Max Birk, and Jan D. Smeddinck. 2015. Designing Wheelchair-Based Movement Games. ACM Transactions on Accessible Computing (TACCESS) 6, 2 (March 2015), Article No. 6. http://dx.doi.org/10.1145/2724729

12. Kathrin M. Gerling, Matthew Miller, Regan L. Mandryk, Max V. Birk, and Jan D. Smeddinck. 2014. Effects of Balancing for Physical Abilities on Player Performance, Experience, and Self-Esteem in Exergames. In: Proceedings of the SIGCHI Conference on Human Factors in Computing Systems (CHI '14), 2201-2210. http://dx.doi.org/10.1145/2556288.2556963

13. Kathrin M. Gerling, Conor Linehan, Ben Kirman, Michael R. Kalyn, Adam B. Evans, and Kieran C. Hicks. 2015. Creating Wheelchair-Controlled Video Games: Challenges and Opportunities when Involving Young People with Mobility Impairments and Game Design Experts. International Journal of HumanComputer Studies. http://dx.doi.org/10.1016/j.ijhcs.2015.08.009

14. Mona L. Guha, Allison Druin, and Jerry A. Fails. 2008. Designing with and for children with special needs: An inclusionary model. In: Proceedings of the $7^{\text {th }}$ International Conference on Interaction Design and Children (IDC '08), 61-64. http://dx.doi.org/10.1145/1463689.1463719

15. Hamilton A. Hernandez, Zi Ye, T.C. Nicholas Graham, Darcy Fehlings, and Lauren Switzer. 2013. Designing Action-based Exergames for Children with Cerebral Palsy. In: Proceedings of the SIGCHI Conference on Human Factors in Computing Systems (CHI '13), 1261-1270. http://dx.doi.org/10.1145/2470654.2466164

16. Harald Holone and Jo Herstad. 2013. Three Tensions in Participatory Design for Inclusion. In: Proceedings of the SIGCHI Conference on Human Factors in Computing Systems (CHI '13), 2903-2906. http://dx.doi.org/10.1145/2470654.2481401

17. IGDA Game Accessibility Guidelines. 2015. Retrieved August 8, 2015 from http://igda-gasig.org/about-gameaccessibility/development-frameworks/

18. Rilla Khaled and Asimina Vasalou. 2014. Bridging Serious Games and Participatory Design. International Journal of Child-Computer Interaction 2, 2 (May 2014), 93-100. http://dx.doi.org/10.1016/j.ijcci.2014.03.001 
19. Gillian King, Theresa Petrenchik, Mary Law, and Patricia Hurley. 2009. The Enjoyment of Formal and Informal Recreation and Leisure Activities: A comparison of school-aged children with and without physical disabilities. International Journal of Disability, Development and Education 56, 2 (April 2009), 109-130.

20. Laura Malinverni, Joan Mora-Guiard, Vanesa Padillo, Maria-Angeles Mairena, Amaia Hervás, and Narcis Pares. 2014. Participatory Design Strategies to Enhance the Creative Contribution of Children with Special Needs. In: Proceedings of the 2014 Conference on Interaction Design and Children (IDC '14), 85-94. http://dx.doi.org/10.1145/2593968.2593981

21. David T. Mitchell and Sharon L. Snyder. 2001. The Uneasy Home of Disability in Literature and Film. In: Gary L. Albrecht, Katherine D., Seelman, and Michael Bury (Eds.): Handbook of Disability Studies. Sage Publications: Thousand Oaks, CA, USA.

22. Tony Morelli and Eelke Folmer. 2014. Real-time sensory substitution to enable players who are blind to play video games using whole body gestures. Entertainment Computing 5, 1 (January 2014), 83-90. http://dx.doi.org/10.1016/j.entcom.2013.08.003

23. Michael J. Muller and Allison Druin.2009. Participatory Design: The Third Space in HCI. In: Andrew Sears and Julie A. Jacko (Eds.): HumanComputer Interaction. CRC Press: Boca Raton.

24. Thomas J. O'Connor, Shirley G. Fitzgerald, Rory A. Cooper, Tricia A. Thorman, and Michael L. Boninger. 2001. Does computer game play aid in motivation of exercise and increase metabolic activity during wheelchair ergometry? Medical Engineering \& Physics 23, 4 (May 2001), 267-273. http://dx.doi.org/10.1016/S1350-4533(01)00046-7

25. Luc Pauwels. 2010. Visual Sociology Reframed: An Analytical Synthesis and Discussion of Visual Methods in Social and Cultural Research. Sociological Methods \& Research 38, 4 (May 2010), 545-581.

26. John R. Porter and Julie A. Kientz. 2013. An empirical study of issues and barriers to mainstream video game accessibility. In: Proceedings of the $15^{\text {th }}$ International ACM SIGACCESS Conference on Computers and Accessibility (ASSETS '13), Article No. 3. http://dx.doi.org/10.1145/2513383.2513444

27. Md. A. Rahman, Delwar Hossain, Ahmad M. Qamar, Faizan U. Rehman, Asad H. Toonsi, Mohamed Ahmed, Abdulmotaleb El Saddik, and Saleh Basalamah. 2014. A Low-cost Serious Game Therapy Environment with Inverse Kinematic Feedback for Children Having Physical Disability. In: Proceedings of International Conference on Multimedia Retrieval (ICMR '14), 529. http://dx.doi.org/10.1145/2578726.2582619
28. Kyle Rector, Cynthia L. Bennett, and Julie A. Kientz. 2013. Eyes-free yoga: an exergames using depth cameras for blind \& low vision exercise. In: Proceedings of the $15^{\text {th }}$ International ACM SIGACCESS Conference on Computers and Accessibility (ASSETS '13), Article No. 12. http://dx.doi.org/10.1145/2513383.2513392

29. Diane Schwartz, Elfreda Blue, Mary McDonald, George Giuliani, Genevieve Weber, Holly Seirup, Sage, Rose, Deborah Elkis-Albuhoff, Jeffrey Rosenfeld, and Andrea Perkins. Dispelling stereotypes: promoting disability equality through film. Disability \& Society 25, 7 (November 2010), 841-848.

30. Anne B. Smith. 2007. Children as social actors: An introduction. International Journal of Children's Rights 15, 1(2007), 1-4.

31. Abbie Solish, Adrienne Perry, and Patricia Minnes. 2010. Participation of Children with and without Disabilities in Social, Recreational and Leisure Activities. Journal of Applied Research in Intellectual Disabilities 23, 3 (2010), 226-236.

32. Diane E. Taub and Kimberly R. Greer. 2000. Physical Activity as a Normalizing Experience for School-Age Children with Physical Disabilities. Journal of Sport \& Social Issues 24, 4 (November 2000), 395-414.

33. Jonathan Waddington, Conor Linehan, Kathrin Gerling, Kieran Hicks, and Timothy L. Hodgson. 2015. Participatory Design of Therapeutic Video Games for Young People With Neurological Vision Impairment. In: Proceedings of the $33^{\text {rd }}$ Annual ACM Conference on Human Factors in Computing Systems (CHI '15), 3533-3542. http://dx.doi.org/10.1145/2702123.2702261

34. Thomas Westin, Kevin Bierre, Dimitris Gramenos, and Michelle Hinn. 2010. Advances in Game Accessibility from 2005 to 2010. Universal Access in HumanComputer Interaction, 400-409.

35. Jacob O. Wobbrock, Shaun K. Kane, Krzysztof Z. Gajos, Susumu Harada, and Jon Froehlich. 2011. Ability-Based Design: Concept, Principles, and Examples. ACM Transactions on Accessible Computing (TACCESS) 3, 3 (April 2011), Article No. 9. http://dx.doi.org/10.1145/1952383.1952384

36. Peter Wright and John McCarthy. 2008. Empathy and Experience in HCI. In: Proceedings of the SIGCHI Conference on Human Factors in Computing Systems (CHI '08), 637-646. http://dx.doi.org/10.1145/1357054.1357156

37. Bei Yuan, Eelke Folmer, and Frederick C. Harris, Jr. 2011. Game accessibility: a survey. Universal Access in the Information Society 10, 1 (March 2011), 81-100. http://dx.doi.org/10.1007/s10209-010-0189-5 